\title{
Mirror symmetry and actions of braid groups on derived categories
}

\author{
R. P. Thomas
}

\begin{abstract}
After outlining the conjectural relationship between the conjectural mirror symmetry programmes of Kontsevich and Strominger-Yau-Zaslow, I will describe some natural consequences of this which are proved from scratch in joint work with Mikhail Khovanov and Paul Seidel. Namely, actions of braid groups are found on derived categories of coherent sheaves, dual to Seidel's braid group of symplectic automorphisms ("generalised Dehn twists") of the mirror. These Fourier-Mukai transforms, one for every "spherical" element of the derived category (a simple rigid sheaf on a Calabi-Yau 3-fold is an example) are closely related to the "mutations" of exceptional bundles on Fanos. Examples of conjecturally mirror dual group actions on triangulated categories are drawn from smoothings and resolutions of 2 and 3 dimensional singularities.
\end{abstract}

\section{Introduction}

In this talk I will attempt to do two things: review a conjectural picture of mirror symmetry, which will no doubt crop up in many other talks, and explain some of its consequences which are proved (independently of the conjectures) in joint work with Mikhail Khovanov and Paul Seidel [ST, [KS]. This is not the mirror symmetry of Gromov-Witten invariants and variations of Hodge structure (yet) but the more fundamental programmes of Kontsevich [K] and StromingerYau-Zaslow [SYZ] which should eventually lead back to the traditional predictions of mirror symmetry.

Section 3 explains the two programmes and their supposed link in the language of Fourier-Mukai transforms, which are reviewed and explained in Section 2. Though many of the objects used in the models are yet to be defined, we can attempt to deduce some consequences and prove these. In particular, symplectomorphisms of a Calabi-Yau manifold should induce autoequivalences of the bounded derived category $D^{b}(X)$ of coherent sheaves on the mirror $X$. We find many such autoequivalences, and actions of the braid group on $D^{b}(X)$ in particular cases (such as on resolutions of singularities). These should be mirror dual to braid groups of symplectomorphisms that arise from configurations of Lagrangian spheres. These spheres are often the vanishing cycles of the smoothing of another, dual, singularity, and we discuss this mirror symmetry of singularities. In Section 5 we mention

1991 Mathematics Subject Classification. Primary 18-06, 18E30; Secondary 14F05, 58F05, 20F36.

The author is supported by Hertford College, Oxford. 
briefly the relationship of this work to mutations of bundles on Fano manifolds, and finally in Section 6 we outline the faithfulness of the braid group action.

Acknowledgements. My main debts are to Mikhail Khovanov and Paul Seidel who allowed me to talk about this work as if it were my own. Kontsevich and Bridgeland and Maciocia have also discussed the twist (4.1). There are many more acknowledgements in [ST]. I would also like to thank the organisers of the 1999 Harvard Winter School on Mirror Symmetry for inviting me to take part.

\section{Fourier-Mukai transforms}

Function transforms. We begin by introducing Fourier-Mukai transforms by analogy with function transforms. Suppose we are given a family $F_{p}$ of (complexvalued, say) functions or distributions on some space $V$, parametrised by some dual space $\widehat{V} \ni p$.

Let $F: V \times \widehat{V} \rightarrow \mathbb{C}$ be the universal function (with $\left.F\right|_{V \times\{p\}}=F_{p}$ ). For instance we could take $V=\mathbb{R}^{n}, \widehat{V}=\left(\mathbb{R}^{n}\right)^{*}$ with $F(x, p)=e^{i p . x}=F_{p}(x)$. Similarly on any manifold $V$ we can take $\widehat{V}=V$ and $F=\delta_{\Delta}$, the Dirac-delta of the diagonal $\Delta$, with $F_{p}(x)=\delta_{p}(x)$.

Suppose now that $\left\{F_{p}: p \in \widehat{V}\right\}$ span (some class of) functions $V \rightarrow \mathbb{C}$, and are orthonormal with respect to some inner product (e.g. $\int F_{p} \bar{F}_{q}=\delta_{p q}$ in a distributional sense). Then for any $f$ in this class, $f$ is built up from the $F_{p} \mathrm{~s}$, with the coefficient of $F_{p}$ being $\widehat{f}(p)=\int_{V} f \bar{F}_{p}$, and we sum $\widehat{f}(p) F_{p}$ over $p$ to regain $f$. That is,

$$
f=\int_{\widehat{V}}\left[\int_{V} f \bar{F}_{p}\right] F_{p} d p
$$

so $f=(\widehat{f})^{\vee}$, where ${ }^{\vee}$ is the dual transform $g^{\vee}(x)=\int_{\widehat{V}} g(p) F(p, x) d p$.

For instance for $F=e^{i p . x}$ we get the Fourier Inversion theorem (though theorem is a little strong for the above treatment) for the Fourier transform ${ }^{\wedge}$ and its inverse $\vee$. Similarly taking $F=\delta_{\Delta}$ on $V \times V$ gives two applications of the identity map: $\widehat{f}(p)=\int_{V} f \delta_{p}=f(p)$.

The way to look at this most relevant to sheaves is via the diagram

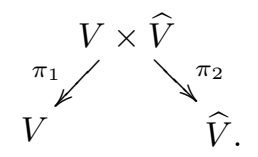

Then the transforms are

$$
\begin{aligned}
\widehat{f} & =\left(\pi_{2}\right)_{*}\left[\pi_{1}^{*} f \cdot \bar{F}\right], \\
g^{\vee} & =\left(\pi_{1}\right)_{*}\left[\pi_{2}^{*} g \cdot F\right] .
\end{aligned}
$$

Thus we pull up a function from $V$ to the product, multiply by $\bar{F}$, and push down the fibres $V_{p}$ of $\pi_{2}\left(\pi_{*}\right.$ means integrate down the fibres of $\left.\pi\right)$ : at each $p$ we integrate $f$ against $\bar{F}_{p}$ to get $\widehat{f}(p)$. Notice also that we can recover the functions $F_{p}$ parametrised by $p$ as the inverse transform of the Dirac-delta $\delta_{p}$,

$$
F_{p}=\delta_{p}^{\vee}
$$

We can also do this in a family. For instance on $V \times Z$ we can carry an extra parameter $z \in Z$ and take the usual transform at each point $z$, i.e. the transform 
just with respect to the variables $x$ and $p$,

$$
f(x, z) \hat{\mapsto} \widehat{f}(p, z) .
$$

This is just a relative transform on $(V \times Z) \times_{Z}(\widehat{V} \times Z)=V \times \widehat{V} \times Z$. Alternatively we can write it as a strict transform on $(V \times Z) \times(\widehat{V} \times Z)$. The function parametrised by $(p, z) \in \widehat{V} \times Z$ is $F_{p} . \delta_{z}$, where $F_{p}$ is (the pull-back to $V \times Z$ of) one of the family of functions on $V$, and $\delta_{z}$ is the Dirac-delta concentrated on $V \times\{z\}$. Again these functions

$$
\left\{F_{p, z}=F_{p} . \delta_{z}:(p, z) \in \widehat{V} \times Z\right\}
$$

form an orthonormal set, giving a transform with universal function $F \delta_{\Delta}$, where $F$ is the pull-back of the universal function on $V \times \widehat{V}$ and $\Delta=V \times \widehat{V} \times Z \subset$ $(V \times Z) \times(\widehat{V} \times Z)$ is the diagonal. The transform $\widehat{f}(p, z)$ is of course the same as (2.3).

Similarly we can have a non-trivial family $X \rightarrow Z$, with dual family $\widehat{X} \rightarrow Z$, and a relative transform on $X \times{ }_{Z} \widehat{X}$ or a strict transform on $X \times \widehat{X}$. Again they are the same, with the universal function on $X \times_{Z} \widehat{X}$ pushed forward via the diagonal map $X \times_{Z} \widehat{X} \hookrightarrow X \times \widehat{X}$ to a function supported on this diagonal. This will seem much more natural in the setting of sheaves.

Sheaf transforms. Suppose now we have a family $F_{p}$ of vector bundles, or coherent sheaves, or complexes of sheaves, on some complex manifold $X$, parametrised by some dual variety $\widehat{X} \ni p$. We will also need to assume the existence of a universal object $F$ on $X \times \widehat{X}$ whose restriction to each $X \times\{p\}$ is $F_{p}$.

We will define the inverse transform first (denoted here by the unfortunate notation ${ }^{\vee}$; this is not the dual of a sheaf but its transform). We would like to think of a sheaf/bundle/complex $\mathcal{F}$ on $\widehat{X}$ as giving a distribution of the $F_{p}$-components of a sheaf $\mathcal{F}^{\vee}$ on $X$, i.e. $\mathcal{F}^{\vee}$ should be the sum over $p \in \widehat{X}$ of the sheaves $\left.\mathcal{F}\right|_{p} \otimes F_{p}$. So with respect to the diagram

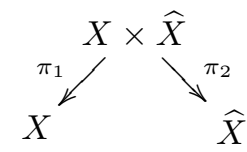

we would like to set, as in (2.1),

$$
\mathcal{F}^{\vee}=\left(\pi_{1}\right)_{*}\left[\left(\pi_{2}^{*} \mathcal{F}\right) \otimes F\right] .
$$

Since a sheaf assigns groups of sections to open sets, the right notion of pushdown $\left(\pi_{1}\right)_{*}$ (the sum of all of the sections over $p \in \widehat{X}$, the analogue of integration down the fibres) is to assign to an open set $U$ all sections on $\pi_{1}^{-1}(U)=U \times \widehat{X}$. In fact numbers of sections down fibres can jump, or be zero, due to the presence of higher cohomology. So we actually take the right derived functor $\mathbf{R}\left(\pi_{1}\right)_{*}$ described in my last talk - this is the complex of sheaves obtained by resolving by injective sheaves and applying $\left(\pi_{1}\right)_{*}$ to this complex. For similar reasons the tensor product should be the derived tensor product $\stackrel{\mathrm{L}}{\otimes}$, and the restriction to fibres $\otimes \mathcal{O}_{X \times\{p\}}$ we have mentioned before should also be taken in this derived sense. 
Thus the correct setting is the bounded derived category of coherent sheaves $D^{b}$, with a universal object $F \in D^{b}(X \times \widehat{X})$. We set, for $\mathcal{F} \in D^{b}(\widehat{X})$,

$$
\mathcal{F}^{\vee}=\mathbf{R} \pi_{1 *[}\left[\left(\pi_{2}^{*} \mathcal{F}\right) \stackrel{\mathbf{L}}{\otimes} F\right]
$$

in most of the simple cases we shall be interested in one can think of vector bundles rather than complexes of sheaves, and normal pushdown and tensor product.

For instance the inverse transform of the structure sheaf $\mathcal{O}_{p}$ of a point $p \in \widehat{X}$ is

$$
\mathcal{O}_{p}^{\vee}=\mathbf{R} \pi_{1 *}\left[\mathcal{O}_{X \times\{p\}} \stackrel{\mathbf{L}}{\otimes} F\right]=\pi_{1 *} F_{p}=F_{p},
$$

the (derived) restriction of $F$ to $X \times\{p\}$, i.e. the object of $D^{b}(X)$ parametrised by $p \in \widehat{X}$; compare (2.2). Similarly the inverse transform of $\bigoplus_{i} \mathcal{O}_{p_{i}}$ is $\bigoplus_{i} F_{p_{i}}$.

The transform from $D^{b}(X)$ to $D^{b}(\widehat{X})$ is given by the formula (c.f. $(2.1)$ )

$$
\widehat{-}=\mathbf{R} \pi_{2 *}\left[\left(\pi_{1}^{*}(\cdot) \stackrel{\mathbf{L}}{\otimes} F^{*}\right][n],\right.
$$

where here we have denoted by $F^{*}$ the derived dual of $F, \mathbf{R} \mathcal{H o m}\left(F, \mathcal{O}_{X \times \widehat{X}}\right)$, and $n=\operatorname{dim} X$. Thus for $\mathcal{F} \in D^{b}(X)$ we have

$$
\widehat{\mathcal{F}}=\mathbf{R} \pi_{2 *}\left[\mathbf{R} \mathcal{H o m}\left(F, \pi_{1}^{*} \mathcal{F}\right)\right][n] .
$$

For $\vee$ and ${ }^{\wedge}$ to be actual inverses (equivalences is the correct categorical notion) we need the objects $F_{p}=F \stackrel{\mathbf{L}}{\otimes} \mathcal{O}_{X \times\{p\}}$ to be orthogonal [B],

$$
\mathbf{R} \operatorname{Hom}\left(F_{p}, F_{q}\right) \cong 0, \quad p \neq q,
$$

orthonormal, in the sense that they are simple,

$$
\operatorname{Hom}\left(F_{p}, F_{p}\right)=\mathbb{C} \cdot \mathrm{id}
$$

and to satisfy the dimension and partial Calabi-Yau conditions $\mathbf{M a}$

$$
\begin{aligned}
\operatorname{dim} X & =\operatorname{dim} \widehat{X} \\
F_{p} \otimes \omega_{X} & \cong F_{p} \quad \forall p .
\end{aligned}
$$

Recently Bridgeland $[\overrightarrow{B r}]$ has shown that for $X$ and $\widehat{X}$ smooth projective varieties these conditions (2.6), (2.7), (2.8) are also sufficient for the Fourier-Mukai transform given by $F \in D^{b}(X \times \widehat{X})$ to be an equivalence. Here $\omega_{X}$ is the canonical bundle of $X$, and we are requiring this to be trivial on the support of each $F_{p}$; recall that $X$ is Calabi-Yau if $\omega_{X}$ is globally trivial.

ExAmple 2.1. Take $\widehat{X}=X$ and $F=\mathcal{O}_{\Delta}$, the structure sheaf of the diagonal parametrising the sheaves $F_{x}=\mathcal{O}_{x}$. Then the transform is the identity, and the partial Calabi-Yau condition (2.8) is vacuous since $\mathcal{O}_{x} \otimes \omega_{X}$ is trivially isomorphic to $\mathcal{O}_{x}$.

ExAmPle 2.2. The original Fourier-Mukai transform [Mu. Let $T$ be an elliptic curve, and let $\widehat{T} \ni t$ be its Jacobian parametrising degree 0 line bundles $L_{t}$ on $T$. We take $F=L$ to be a Poincaré line bundle on $T \times \widehat{T}$ (for instance if we fix a basepoint $t_{0} \in T$ then we can identify $T$ with $\widehat{T}$, and $L$ is given by the divisor $\left.\Delta-\left(\left\{t_{0}\right\} \times T\right) \subset T \times T\right)$. 
Then the $L_{t}$ s form an orthonormal set:

$$
H^{0}\left(L_{s}^{*} \otimes L_{t}\right)=H^{1}\left(L_{s}^{*} \otimes L_{t}\right)= \begin{cases}\mathbb{C} & s=t \\ 0 & s \neq t\end{cases}
$$

So as $T$ is Calabi-Yau we get an invertible transform

$$
\mathcal{F} \mapsto \widehat{\mathcal{F}}=\mathbf{R} \pi_{1 *}\left[L \otimes \pi_{2}^{*} \mathcal{F}\right]
$$

with fibre over the generic point $t \in \widehat{T}$ (at which base-change holds) " $H^{0}(\mathcal{F} \otimes$ $\left.L_{t}\right)-H^{1}\left(\mathcal{F} \otimes L_{t}\right)$ " (really a complex with these two cohomologies). Thus, when $H^{1}$ vanishes, we replace $\mathcal{F}$ by its spectrum of sections $\left\{H^{0}\left(\mathcal{F} \otimes L_{t}\right): t \in \widehat{T}\right\}$ of different twists. The inverse transform uses the dual line bundle, and the set-up is symmetric between $T$ and $\widehat{T}$.

Then using (2.5) we see that the transform of the structure sheaf $\mathcal{O}_{t}$ of a point $t \in \widehat{T}$ is the corresponding line bundle $L_{t}$, while $r$ points transform to the appropriate rank $r$ bundle (which is the sum of $r$ line bundles). We depict this as follows, drawing a basis of the fibre of the vector bundle over the right hand torus.

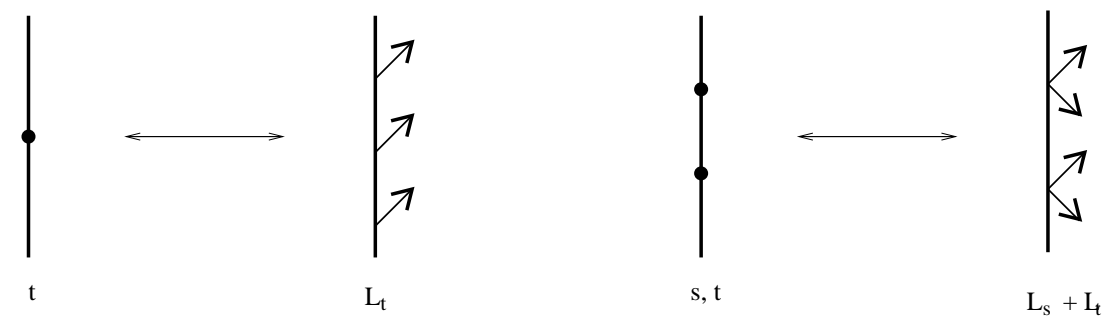

Thus, inversely, we have an algebraic gadget that takes a degree 0 semistable bundle on $T$ and splits it into its constituent pieces according to Atiyah's classification; for every $L_{t}$ factor we get a point $t \in \widehat{T}$. The non-trivial extensions correspond to structure sheaves of double points, etc.

Similarly we can do this in a family. Consider an elliptic fibration $X \rightarrow Z$, possibly with singular fibres. Suppose we also have a section $s$ so that we can identify $X$ with its relative Jacobian. Then there is a Poincaré sheaf $\mathcal{L}$ on $X \times_{Z} X$ corresponding to the Weil divisor $\Delta-s(Z) \times_{Z} X$. Rather than doing a relative transform on this singular space, we do a strict transform on $X \times X$ by pushing forward $\mathcal{L}$ to $\iota_{*} \mathcal{L}$ via $\iota: X \times_{Z} X \rightarrow X \times X$. Then setting

$$
\widehat{\mathcal{F}}=\mathbf{R} \pi_{1 *}\left[\iota_{*} \mathcal{L} \otimes \pi_{2}^{*} \mathcal{F}\right]
$$

we get a transform $D^{b}(X) \rightarrow D^{b}(X)$, a family version of the previous example, giving pictures like:

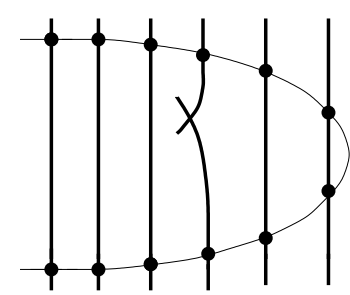

Multisection $C$ ( + line bundle on it $) \quad \longleftrightarrow \quad$ Vector bundle, deg 0 on fibres 
where the covering degree $r$ of the multisection (its intersection with the fibre) is the rank $r$ of the vector bundle.

Thus vector bundles correspond to "spectral covers" in the usual way - the cover gives $r$ points on each fibre yielding the sum of $r$ line bundles on the dual fibre. Globally we patch these together in the base direction using the line bundle on $C$, and get a non trivial vector bundle which is not globally a sum of line bundles (because $C$ is not globally $r$ disjoint sections).

The rigorous statement is that there is an autoequivalence of $D^{b}(X)$ given by this transform. It is invertible since the universal sheaf $\iota_{*} \mathcal{L}$ is concentrated on torus fibres, about which $X$ is locally Calabi-Yau so that $\iota_{*} \mathcal{L} \otimes \omega_{X} \cong \iota_{*} \mathcal{L}$. For us, the point is simply that there is an algebraic gadget that converts spectral covers like $C$, with line bundles on them, into vector bundles (degree 0 and semistable on the fibres), and vice-versa. It does this for free, without any analysis of the singular fibres, since $X \times X$ is smooth (even though $X \times_{Z} X$ is not). This is at the expense of introducing the derived category, of course, but as we see here in many cases the transform takes sheaves to sheaves instead of a complex of sheaves.

\section{Kontsevich vs. Strominger-Yau-Zaslow}

We now turn to mirror symmetry and the two competing conjectural theories. Strominger-Yau-Zaslow [SYZ] suggest that (in an appropriate complex structure) a Calabi-Yau $n$-fold $X$ should admit a fibration by special Lagrangian tori $\left(T^{n} \hookrightarrow X\right.$ is Lagrangian if the symplectic (Kähler) form restricts identically to zero on $T^{n}$, and special if the restriction of the imaginary part of the Calabi-Yau $(n, 0)$-form is identically zero) with a special Lagrangian section. In this case the mirror $\widehat{X}$ should be the dual torus fibration.

Kontsevich [|K], on the other hand, conjectures that there should be a natural exact equivalence of triangulated categories (an exact equivalence is one which preserves the distinguished triangles) between $D^{b}(X)$ and $D^{b}(\operatorname{Fuk}(\widehat{X}))$. The second category here is the derived category of the Fukaya category of the symplectic manifold $\widehat{X}$, and as such is not yet properly constructed (though see Fukaya's talk for more progress on this). Other talks will explain more about the Fukaya category; all we need to know is that it is constructed from only the symplectic geometry of $\widehat{X}$, using (graded) Lagrangian submanifolds, local systems on them, and their Fukaya-Floer homology. In particular every Lagrangian cycle $L$ in $\widehat{X}$ (plus a grading), with a flat line bundle on it, should give an object in $D^{b}(\operatorname{Fuk}(\widehat{X}))$. The corresponding object $\mathcal{F}_{L}$ in $D^{b}(X)$ should have the same Homs, so that $H F^{*}(L, L)$ should be quasi-isomorphic to $\mathbf{R} \operatorname{Hom}\left(\mathcal{F}_{L}, \mathcal{F}_{L}\right)$.

The (again conjectural) correspondence between the two pictures is now folklore and has been discussed by many people, see for instance [AP, $\mathbf{T y}]$. The basic idea is that a Lagrangian multisection $L$ in the fibration $\widehat{X}$ (with a flat line bundle on it, and intersection with the fibre $r$ ) should correspond to a rank $r$ holomorphic vector bundle on $X$ by an analytical version of the Fourier-Mukai transform, giving a diagram like the one above. That is, the intersection of $L$ with a fibre gives $r$ points corresponding to $r$ line bundles on the dual torus, as before. Special Lagrangian sections should perhaps correspond to bundles with Hermitian-YangMills connections (i.e. stable bundles) as suggested in $\mathbf{V a}$ (both special and stable are stability conditions on the objects on either side under a natural group action, so this makes sense). 
In 2 complex dimensions we can rigorously carry out this procedure, since we have the tools of algebraic geometry and Fourier-Mukai to deal with the singularities. We also have Yau's solution of the Calabi conjecture, which gives a hyperkähler metric on a compact Calabi-Yau surface (i.e. a $K 3$ or $T^{4}$ ). Thus for every complex structure $I$ there is a conjugate quaternionic partner $J$, and if we rotate the complex structure from $I$ to $J$ then the special Lagrangian cycles become the complex curves on $X$. Thus, after this hyperkähler rotation, the SYZ conjecture is concerned with an elliptically fibred surface with a section, and the mirror should be the dual fibration. Thus in this dimension the mirror is topologically the same as $X$ (in 3 dimensions there is a topology change at the singular fibres) and the correspondence we want is precisely the Fourier-Mukai transform described before. This gives an equivalence of categories $D^{b}(X) \rightarrow D^{b}(X)$, which is the appropriate even dimensional mirror symmetry, taking $0,2,4$ branes (points, 2-cycles, and vector bundles) to even dimensional 0,2,4 branes (3 dimensional mirror symmetry as we have described should take $0,2,4,6$ branes to 3 branes).

We can ask what else we get from the Fourier-Mukai transform. By (2.5) a fibre, or more generally the pushforward of a line bundle on a fibre, gets taken to the corresponding point in the dual fibre:

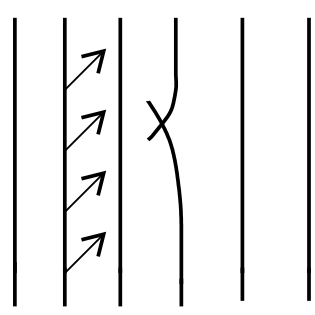

$\mathrm{i}_{*} \mathrm{~L}_{\mathrm{t}}$

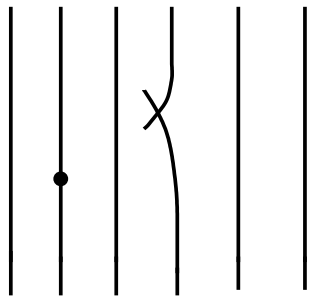

$\mathrm{t}$

This was of course the original idea of SYZ: that the moduli of special Lagrangian tori, plus flat line bundles, on $\widehat{X}$ should be isomorphic to the moduli of points on $X$, i.e. to $X$ itself.

On non-hyperkähler manifolds we cannot deduce anything much about either programme without hard analysis, and that is best left to other speakers here. But we can deduce some consequences, and try to prove these. The surface case which could be dealt with rigorously showed one thing: while the mirror $\widehat{X}$ was isomorphic to the original $X$, the mirror map was certainly not the identity: in fact it took points to fibres plus line bundles, rather than points, and induced a non trivial map on $H^{*}(X)$ on taking Mukai vectors.

In particular we see that mirror symmetry is not functorial on points (a phrase I learnt from Paul Seidel); in fact, as Kontsevich envisaged, (graded) symplectic automorphisms of the mirror $\widehat{X}$ should not induce holomorphic automorphisms of $X$, but autoequivalences of its derived category $D^{b}(X)$.

This is what we shall concentrate on.

\section{Autoequivalences of derived categories and braid groups}

Autoequivalences of derived categories. What are the autoequivalences of $D^{b}(X)$ ? There are the obvious ones given by translation of complexes $[n]$, 
tensoring with line bundles $\otimes L$, and those pulled back from automorphisms of $X$. Bondal and Orlov BO have shown that this is all of them for $X$ smooth and projective with ample canonical bundle or anticanonical bundle. In fact for there to be any more $X$ must be partially Calabi-Yau: Orlov $[\mathrm{Or}]$ has shown that any autoequivalence is set up by an object $F \in D^{b}(X \times \widehat{X})$ on the product as a FourierMukai transform (2.4), which by (2.8) must then satisfy $F_{p} \otimes \omega_{X} \cong F_{p}$ for all $p \in X$. And for Calabi-Yau manifolds Kontsevich's proposal predicts there should be many such Fourier-Mukai transforms; in particular there should be one for every (graded) symplectomorphism of the mirror $\widehat{X}$.

For instance Seidel [S] has shown that given any Lagrangian sphere $L \cong S^{n}$ in a symplectic manifold we may construct a symplectomorphism - the generalised Dehn twist about $L$. This is given as monodromy around a degeneration of the manifold in which the sphere is collapsed to (becomes the vanishing cycle of) an ordinary double point. Alternatively we can glue in the following local model twist on $T^{*} S^{n}$. Give $T^{*} S^{n}$ its standard symplectic structure and metric. Then $\mu(\xi)=|\xi|$, the length of a cotangent vector $\xi$, gives a Hamiltonian function which induces a circle action - the flow from $\xi$ (considered as a tangent vector using the metric) is the unit speed geodesic flow in the direction $\xi$ along $S^{n}$, lifted horizontally to $T S^{n} \cong T^{*} S^{n}$ as $(x(t), \xi(t)=\dot{x}(t)|\xi(0) / \dot{x}(0)|)$. This flow is clearly discontinuous across the zero section $S^{n} \subset T^{*} S^{n}$ (it is unit speed in opposite directions as we pass through the zero section) but since the geodesics have length $2 \pi$, the flow through time $\pi$ is continuous, and gives the antipodal map. So we may define the generalised Dehn twist as the flow of any point $\xi$ through an angle varying smoothly from $0=2 \pi$ as $|\xi| \rightarrow \infty$ to $\pi$ at the zero section $|\xi|=0$; see $[\mathbf{S}]$.

The Dehn twist has a canonical lift to the graded symplectomorphism group of $\widehat{X}$, and so should be dual to a Fourier-Mukai transform constructed from an element $\mathcal{F}_{L}$ of $D^{b}(X)$ (not $D^{b}(X \times X)$, notice) dual to the Lagrangian $L$. Since Homs should be the same on both sides, we know that $\mathbf{R} \operatorname{Hom}\left(\mathcal{F}_{L}, \mathcal{F}_{L}\right)$ should be isomorphic to $H F^{*}(L, L) \cong H^{*}\left(S^{n}\right)$. Thus we might expect to be able to find an invertible Fourier-Mukai transform for every spherical $\mathcal{F} \in D^{b}(X)$, where

Definition 4.1. $\mathcal{F} \in D^{b}(X)$ is $N$-spherical if $\mathbf{R} \operatorname{Hom}(\mathcal{F}, \mathcal{F}) \cong H^{*}\left(S^{N} ; \mathbb{C}\right)$, where $N=\operatorname{dim} X$. That is

$$
\operatorname{Ext}^{i}(\mathcal{F}, \mathcal{F})=\left\{\begin{array}{cc}
\mathbb{C} & i=0, N \\
0 & i \neq 0, N
\end{array}\right.
$$

Thus for instance a simple $(\operatorname{End}(\mathcal{F})=\mathbb{C}$.id $)$, rigid $\left(\operatorname{Ext}^{1}(\mathcal{F}, \mathcal{F})=0\right)$ sheaf $\mathcal{F}$ on a smooth Calabi-Yau 3-fold is 3 -spherical by Serre duality: $\operatorname{Ext}^{i}(\mathcal{F}, \mathcal{G}) \cong$ $\operatorname{Ext}^{3-i}(\mathcal{G}, \mathcal{F})^{*}$.

Definition 4.2. For an $N$-spherical $\mathcal{F} \in D^{b}(X)$ define the twist $T_{\mathcal{F}} \mathcal{G}$ of $\mathcal{G} \in$ $D^{b}(X)$ to be the cone (total complex) of the evaluation map

$$
\mathcal{F} \stackrel{\mathbf{L}}{\otimes} \mathbf{R} \operatorname{Hom}(\mathcal{F}, \mathcal{G}) \rightarrow \mathcal{G} .
$$

Here we should pick suitable resolutions so the above becomes a genuine map of complexes, then take the total complex of this map. This defines $T_{\mathcal{F}} \mathcal{G}$ only up to quasi-isomorphism (cones are not functorial in $\left.D^{b}(X)\right) ; T_{\mathcal{F}}$ can in fact be made into a functor $D^{b}(X) \rightarrow D^{b}(X)$ [ST]. 
So in simple cases $T_{\mathcal{F}} \mathcal{G}$ will be the kernel or cokernel of a map $\mathcal{F} \otimes \operatorname{Hom}(\mathcal{F}, \mathcal{G}) \rightarrow$ $\mathcal{G}$. For $X$ a smooth projective variety, it is easy to see that $T_{\mathcal{F}}$ is the Fourier-Mukai transform given by the object

$$
\left\{\mathbf{R} \mathcal{H o m}\left(\pi_{2}^{*} \mathcal{F}, \pi_{1}^{*} \mathcal{F}\right) \rightarrow \mathcal{O}_{\Delta}\right\}
$$

of $D^{b}(X \times X)$. Here $\pi_{i}$ is projection to the $i$ th factor $X$, the map is restriction to the diagonal $\Delta$ followed by the trace map, and the braces mean we take the cone (total complex) of the map. However, it is more convenient for us to work with general twists in arbitrary derived categories; thus for instance our results apply to non-compact schemes without difficulty.

The categorical equivalent of the partial Calabi-Yau condition $\mathcal{F} \otimes \omega_{X} \cong \mathcal{F}$ is the existence of a functorial duality (Serre duality for us, or a local form of it in the non-compact case) between $\mathbf{R} \operatorname{Hom}(\mathcal{F}, \mathcal{G})$ and $\mathbf{R} \operatorname{Hom}(\mathcal{G}, \mathcal{F})[N]$; equivalently the pairing

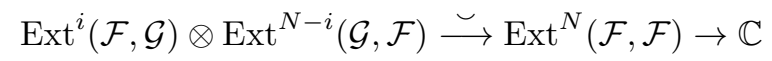

should be perfect, where the last map uses the fact that $\mathcal{F}$ is $N$-spherical.

Definition 4.3. For $\mathcal{F} N$-spherical (4.1) and with a duality (4.3), there is a functor $T_{\mathcal{F}}^{\prime}: D^{b}(X) \rightarrow D^{b}(X)\left[\right.$ ST] such that the quasi-isomorphism class of $T_{\mathcal{F}}^{\prime} \mathcal{G}$ is the total complex we denote

$$
\{\mathcal{G} \rightarrow \mathcal{F} \stackrel{\mathbf{L}}{\otimes} \mathbf{R} \operatorname{Hom}(\mathcal{F}, \mathcal{G})[N]\}
$$

given by dualising a map of chain complexes representing the evaluation

$$
\mathcal{G} \stackrel{\mathbf{L}}{\otimes} \mathbf{R} \operatorname{Hom}(\mathcal{G}, \mathcal{F}) \rightarrow \mathcal{F} .
$$

Again this is really a Fourier-Mukai transform with object the derived dual of (4.2) shifted by $[N]$.

The last three definitions, and the theorems below, can of course be formulated in the derived category of an arbitrary abelian category, linear over a field $k$, having enough injectives, and containing a spherical $\mathcal{F}$ with a duality (4.3). We shall confine ourselves to derived categories of coherent sheaves (this does not have enough injectives and one must work with quasi-coherent complexes with coherent cohomology in the usual way; see [ST] for full details).

Theorem 4.4. For $\mathcal{F}$ spherical with a duality (4.J), $T_{\mathcal{F}}$ and $T_{\mathcal{F}}^{\prime}$ are inverses.

Again we are being sketchy here. The precise statement [ST] takes place in the derived category of a $k$-linear abelian category, and "inverses" means that $T_{\mathcal{F}} \circ T_{\mathcal{F}}^{\prime}$ and $T_{\mathcal{F}}^{\prime} \circ T_{\mathcal{F}}$ are both naturally isomorphic to the identity functor.

Using Serre duality on a compact scheme, or a local form of it one can prove for $\mathcal{F}$ compactly supported on a non-compact scheme, we get an invertible FourierMukai transform in each of the following examples.

- Any simple, rigid sheaf $\mathcal{F}$ on a Calabi-Yau 3-fold $X$. In particular the structure sheaf $\mathcal{O}_{X}$ gives a canonical transform, called the reflection functor by Mukai, which should be mirror dual to the Dehn twist about the (conjectural) special Lagrangian $S^{3}$ zero section of the SYZ fibration of $\widehat{X}$.

- Holomorphic -2-spheres $C$ in a complex surface give Fourier-Mukai transforms, taking $\mathcal{F}=\mathcal{O}_{C}$. In particular we get Fourier-Mukai transforms for general-type surfaces containing such spheres, which might be surprising 
given the results of $[\mathbf{B O}$. These surfaces are locally Calabi-Yau along the spheres. The induced action on cohomology is the Picard-Lefschetz reflection in the corresponding -2 -vector.

- Spheres with normal bundle $\mathcal{O}_{\mathbb{P}^{1}}(-1) \oplus \mathcal{O}_{\mathbb{P}^{1}}(-1)$ in 3 -folds also give transforms in the same way.

- Surfaces $S$ in 3-folds satisfying $h^{0,1}(S)=0=h^{0,2}(S)$, with the local CalabiYau condition $\nu_{S} \cong \omega_{S}$.

Seidel proved in [S] that the Dehn twists along $A_{n}$-chains of Lagrangian spheres satisfy the braid relations. That is, if we have a chain of such spheres with consecutive pairwise intersections one transverse point, then we get a homomorphism from the braid group on $(n+1)$ strands $B_{n+1}$ into the symplectomorphism group of the ambient manifold. Moreover he showed the smoothing of an $A_{n}$ singularity on a complex surface (such as the smoothing of the standard $S L(2, \mathbb{C})$ quotient singularity $\mathbb{C}^{2} / \mathbb{Z}_{n}$ ) contains such a chain of Lagrangian spheres.

For two Lagrangians $L_{i}$ intersecting transversely in a single point we have $H F^{*}\left(L_{1}, L_{2} ; \mathbb{C}\right) \cong \mathbb{C}$, so we define

Definition 4.5. A sequence of spherical objects $\mathcal{F}_{i} \in D^{b}(X), i=1, \ldots, n$ form an $A_{n}$-chain if they satisfy

$$
\sum_{k} \operatorname{dim} \operatorname{Ext}^{k}\left(\mathcal{F}_{i}, \mathcal{F}_{j}\right)= \begin{cases}0 & |i-j|>1 \\ 1 & |i-j|=1\end{cases}
$$

Thus there are no Homs between distinct $F_{i}$ s unless they are consecutive, in which case there is a unique map (up to scale) in some (arbitrary) degree.

THEOREM 4.6. Given an $A_{n}$-chain of spherical objects $\mathcal{F}_{i} \in D^{b}(X)$ with duality (4.3) there are the following natural isomorphisms between the corresponding functors $T_{i}=T_{\mathcal{F}_{i}}$

$$
\begin{array}{ll}
T_{i} T_{i}^{\prime} \cong \mathrm{id} \cong T_{i}^{\prime} T_{i} & \\
T_{i} T_{j} T_{i} \cong T_{j} T_{i} T_{j} & |i-j|=1 \\
T_{i} T_{j} \cong T_{j} T_{i} & |i-j|>1 .
\end{array}
$$

Thus they define a weak braid group action on $D^{b}(X)$. (We have not checked if the natural isomorphisms above satisfy the coherence relations of [De] to define a genuine $B_{n+1}$ action on the category $D^{b}(X)$.)

Thus we get braid group actions on derived categories of coherent sheaves in the following cases.

- $A_{n}$-chains of -2 -spheres $C_{i}$ (i.e. a sequence of -2 -spheres with consecutive pairwise intersections a reduced point) in quasi-projective surfaces give actions of $B_{n+1}$ with $\mathcal{F}_{i}=\mathcal{O}_{C_{i}}$. For instance the ALE spaces that are the resolutions of the $S L(2, \mathbb{C})$ quotient singularities $\mathbb{C}^{2} / \mathbb{Z}_{n}$.

- If, as in the previous example, we have an $A_{n}$-chain of -2 -spheres $C_{i}$, we can twist instead by the corresponding line bundles $L_{i}=\mathcal{O}\left(C_{i}\right)$. Simple exact sequences show that these also form an $A_{n}$-chain of spherical objects in $D^{b}(X)$ for $X$ a $K 3$ surface.

- Chains of surfaces $S_{i}$ in 3-folds, each with $\nu_{S} \cong \omega_{S}$ and $h^{0,1}(S)=0=$ $h^{0,2}(S)$, give braid group actions (for $\mathcal{F}_{i}=\mathcal{O}_{S_{i}}$ ) if $S_{i} \cap S_{j}=\emptyset$ for $|i-j|>1$ and $S_{i} \cap S_{i+1}$ is a $\mathbb{P}^{1}$-fibre of one surface and a -2 -sphere in the other. Again such configurations arise in crepant resolutions of 3 -fold singularities. 
- Taking $\mathcal{F}_{i}$ to be line bundles on an elliptic curve, with the degrees of $\mathcal{F}_{i}$ and $\mathcal{F}_{j}$ differing by $i-j$, we recover the original Fourier-Mukai transforms of $[\mathbf{M u}]$, and from any two consecutive such line bundles we get an action of $B_{3}$, a central extension of $S L(2, \mathbb{Z})$. The central generator $\left(T_{1} T_{2}\right)^{6}$ acts as the translation [2] and the action is easily seen to be the $S L(2, \mathbb{Z})$ action of Mukai. There are mirror, symplectic analogues of these relations for Dehn twists on tori in [ST].

Singularities. Notice the chains of -2 -spheres in surfaces are different in the holomorphic and symplectic cases - the former appear in resolutions of singularities, the latter in smoothings of the same singularities. Are the corresponding braid group actions, on $D^{b}(X)$ and $D^{b}(\mathrm{Fuk}(X))$ respectively, mirror dual?

In the compact case we know what the mirror to the Lagrangian spheres should be in the presence of an SYZ fibration - the Fourier-Mukai transforms of their structure sheaves. If the spheres lie in the elliptic fibres then their transforms are themselves, and the braid group action on the symplectic side should be dual to the first example listed above. If however the spheres are sections of the fibration then their transforms are given by the line bundles $\mathcal{O}\left(C_{i}\right)$ (twisted by $\mathcal{O}(-s)$, where $s$ is the image of the zero section, but this need not concern us), and the correct dual is the second example in the above list.

Either way mirror symmetry seems rather local in these cases, and there are other cases where the mirror dual of the (symplectic) smoothing of one singularity is the (holomorphic) resolution of another. In fact this is the general proposal of Mo: Morrison suggests that moving towards the discriminant locus in the complex structure moduli space of $X$ (i.e. degenerating $X$ to a singular Calabi-Yau) should be mirror dual to moving to a "boundary wall" of the complexified Kähler cone of $\widehat{X}$ (the annihilator of a face of the Mori cone), thus inducing an extremal contraction of $\widehat{X}$. Resolving the singularities of $X$ should then be mirror dual to smoothing the contracted $\widehat{X}$. In particular, in some generic situations, the smoothing of ordinary double points (with their Lagrangian $S^{3}$ vanishing cycles and corresponding Dehn twists on $\widehat{X}$ ) should be mirror to small resolutions of other ordinary double points (with exceptional $\mathbb{P}^{1}$ loci giving corresponding twists $T_{\mathcal{O}_{\mathbb{P} 1}}$ on $D^{b}(X)$ ).

Our results indicate that it might be reasonable to expect certain additional properties of singularities that are dual in this way. Namely, singularities whose smoothings have a Dynkin diagrams of Lagrangian $S^{3}$ vanishing cycles should be dual to singularities whose resolution has a similar diagram of (spherical structure sheaves of) irreducible components of its exceptional set (these form the nodes of the diagram, edges are provided by $\mathbf{R} H o m \mathrm{~s}$ ).

For instance consider the smoothing of the 3-fold singularity

$$
x^{2}+y^{2}+z^{2}+t^{2 n}=0,
$$

which contains an $A_{2 n-1}$-chain of Lagrangian $S^{3}$ vanishing cycles of the singularity giving a braid group of (graded) symplectomorphisms. Peturbing this into $n$ ordinary double points $x^{2}+y^{2}+z^{2}+t^{2}=0$ (which we can do on the symplectic side, we are only varying complex structure), analysing the effect on homology and its mirror, and using Morrison's proposal, we are led in [ST] to ask whether the mirror should be given by the following geometry, which we know leads to a braid group action. 
Proposition 4.7. Suppose we have a chain of Fano surfaces $\left\{S_{2 k}\right\}_{k=1}^{n-1}$ in a smooth 3-fold $X$, with the local Calabi-Yau condition that their normal bundles are isomorphic to their canonical bundles. Suppose also that each $S_{2 k}$ contains two disjoint $(-1)-\mathbb{P}^{1} s, C_{2 k-1}$ and $C_{2 k+1}$, in which it intersects $S_{2 k-2}$ and $S_{2 k+2}$ respectively (and there are no more intersections, so only consecutive surfaces intersect). Then the sheaves

$$
\mathcal{F}_{2 k}=\mathcal{O}_{S_{2 k}} \quad \text { and } \quad \mathcal{F}_{2 k+1}=\mathcal{O}_{C_{2 k+1}}
$$

form an $A_{2 n-1}$-chain, and so define an action of $B_{2 n}$ on $D^{b}(X)$.

In particular if we take the surfaces $S_{2 k}$ to be $\mathbb{P}^{2} \mathrm{~s}$ blown up in two points (giving the two exceptional curves $C_{2 k-1}$ and $C_{2 k+1}$ which we think of as the mirrors of the $(2 k-1)$ th and $(2 k+1)$ th vanishing cycles in the smoothing of $x^{2}+y^{2}+z^{2}+t^{2 n}=0$ according to Morrison's proposal) then there is an extra (-1)-curve $C_{2 k}$ in $S_{2 k}$ - the proper transform of the line joining the two blow-up points - which we can think of as the mirror of the $2 k$ th vanishing cycle. Such a configuration is easily shown ST to arise in smooth toric Calabi-Yau manifolds as the crepant resolution of a nasty singularity that we would like to think of as the dual of $x^{2}+y^{2}+z^{2}+t^{2 n}=0$.

Another relevant example is Arnold's strange duality (see for instance [|Pi]), which is encompassed by mirror symmetry for $K 3$ surfaces according to the work of a number of people (Aspinwall and Morrison, Kobayashi, Dolgachev, Ebeling, etc.). To every isolated surface singularity on Arnold's list, described by three numbers $b_{1}, b_{2}, b_{3}$, there is a natural $K 3$ compactification $S$ of the singularity containing at infinity a chain of -2 -spheres with intersection configuration given by the following Dynkin diagram $T\left(b_{1}, b_{2}, b_{3}\right)$ :

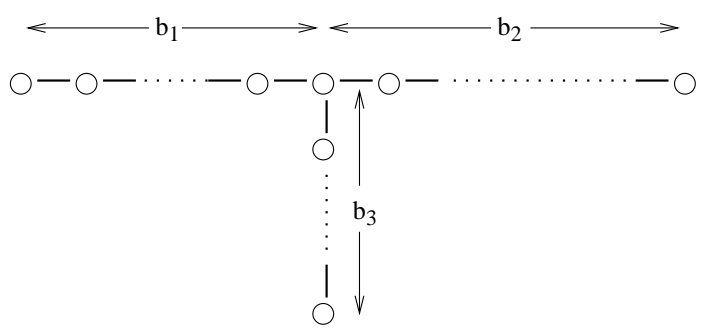

Here the circles represent -2-spheres, and edges give intersections of the spheres of intersection number 1 , and the central -2 -sphere is counted in each $b_{i}$. The corresponding intersection matrix is not negative definite for the numbers $b_{i}$ in the list, so the spheres cannot be completely contracted, though they can be contracted to a smooth $\mathbb{P}^{1}$ with three points on it that are singular points of the surface (this surface is Pinkham's original compactification of the affine surface singularity).

Let $\left\{c_{i}\right\}_{i=1}^{3}$ be the numbers dual to the $b_{i} \mathrm{~s}$ in strange duality (i.e. these are the $b_{i}$ s associated to the dual singularity). Then the intersection matrix of the smoothing of the original singularity has vanishing cycles given by $T\left(c_{i}\right) \oplus H$ (where $H$ is the hyperbolic $\left.\left(\begin{array}{ll}0 & 1 \\ 1 & 0\end{array}\right)\right)$. Together the -2 -spheres plus the vanishing cycles give all of the homology of the smoothed $K 3$ surface: $H_{2}(K 3) \cong T\left(b_{i}\right) \oplus T\left(c_{i}\right) \oplus H$. The duality swaps the $b_{i}$ s and $c_{i}$ s, taking the homology $T\left(c_{i}\right) \oplus H$ generated by the vanishing cycles to the holomorphic homology $T\left(c_{i}\right) \oplus H$ in the mirror $K 3$ (given by the resolution cycles and the hyperbolic $\left.H_{0} \oplus H_{4}\right)$. 
Thus we can think of mirror symmetry as replacing the smoothing of one singularity by the resolution of another (though it is not an isolated singularity, it is the $\mathbb{P}^{1}$ with 3 surface singularities on it). Here we are not thinking of the duality as merely swapping Arnold's singularities, but as a rather more global phenomenon on $K 3$ surfaces, which therefore has to include the chains of -2 -spheres at infinity.

There is a generalised braid group action on the derived category of the $K 3$ surface given by the $T\left(b_{1}, b_{2}, b_{3}\right)-2$-sphere configuration. Dually, there is probably the same group of Dehn twists around the Lagrangian vanishing cycles of the mirror $K 3$, though it seems not to be known whether they can be found in the geometric intersection configuration $T\left(b_{1}, b_{2}, b_{3}\right)$ (they may have many more geometric intersections than their topological intersections suggest).

\section{Mutations}

The formula (4.1) for our twist is familiar to algebraists, in tilting theory, and those who work on exceptional bundles on Fano manifolds - see e.g. [Ru. There the twists are called mutations, and act on certain modules over algebras (similar to those described in the next section) built from the bundles (they cannot give equivalences of derived categories by the result of $[\mathbf{B O} \mid$ ). The bundles $\mathcal{F}$ that one twists are also those with minimal $\operatorname{Ext}^{*}(\mathcal{F}, \mathcal{F})$; in the case of Fano manifolds this means $\mathcal{F}$ is simple and has no higher Exts at all, and is called exceptional.

There are braid group actions of such twists on exceptional collections of bundles, but the relation with our work is far from clear, and it is possible there is none. Here we shall simply note a relationship between exceptional objects on Fano manifolds and spherical objects on Calabi-Yaus, motivated by two examples of $\mathrm{Ku}$.

Definition 5.1. We say that a map $f: X \rightarrow Y$ from a Calabi-Yau $N$-fold $X$ to a smooth projective Fano $M$-fold $Y$, of codimension $c=N-M$ (which may be of any sign or zero), is simple if $\mathbf{R} f_{*} \mathcal{O}_{X}$ is made up from $\mathcal{O}_{Y}$ and $\omega_{Y}$ in the sense that

$$
\begin{array}{ll}
\text { - } c>0 & R^{i} f_{*} \mathcal{O}_{X}=\left\{\begin{array}{cl}
\mathcal{O}_{Y} & i=0 \\
0 & i \neq 0, c \\
\omega_{Y} & i=c
\end{array}\right. \\
\text { - } c=0 & R^{i} f_{*} \mathcal{O}_{X}=\left\{\begin{array}{cl}
\mathcal{O}_{Y} \oplus \omega_{Y} & i=0 \\
0 & i \neq 0
\end{array}\right. \\
\text { - } c<0 & \text { There is an exact triangle } \omega_{Y}[-c] \rightarrow \mathbf{R} f_{*} \mathcal{O}_{X} \rightarrow \mathcal{O}_{Y} .
\end{array}
$$

Simple examples of maps $f$ are often of this type. For instance, for CalabiYaus fibred over a Fano base with generic fibre $F$ such that $h^{0, i}(F)=0,0<i<c$, relative Serre duality shows that the projection is simple in this sense.

Examples with $c=0$ are given by Calabi-Yaus double covering Fanos, branched over a double anticanonical divisor, while for $c=-1$ we have a Calabi-Yau anticanonical divisor in a Fano manifold.

Theorem 5.2. Suppose $f: X \rightarrow Y$ is a simple map, as defined above, and $\mathcal{F} \in D^{b}(Y)$ is exceptional $\left(\operatorname{Ext}^{i}(\mathcal{F}, \mathcal{F})=\mathbb{C}\right.$ for $i=0$, and 0 for $\left.i \neq 0\right)$. Then $\mathbf{L} f^{*} \mathcal{F} \in D^{b}(X)$ is spherical.

In the other direction, i.e. maps from a Fano to a Calabi-Yau, something can only be said in the case of a Fano divisor in a (locally) Calabi-Yau manifold. Namely, 
TheOREM 5.3. Suppose that $\iota: Y \subset X$ is a smooth Fano divisor with normal bundle $\nu_{Y}=\omega_{Y}$ in a quasi-projective scheme $X$. If $\mathcal{F} \in D^{b}(Y)$ is exceptional then $\iota_{*} \mathcal{F} \in D^{b}(X)$ is spherical.

This takes care of most of the examples of spherical sheaves given until now (by taking the exceptional sheaf to be $\mathcal{O}_{Y}$ ), and provides many more by pushing forward the exceptional collections of [ $[\mathbf{R u}]$ to (local) Calabi-Yau manifolds.

\section{Fidelity}

Finally we briefly mention what is in many ways the main result of $[\mathbf{K S}, \mathbf{S T}]$, namely that in dimension $N \geq 2$, the $B_{n+1}$ actions given by $A_{n}$-chains of spherical objects are faithful.

To do this it is clearly enough to show the induced $B_{n+1}$ action on the differential graded modules $\bigoplus_{i} \mathbf{R} \operatorname{Hom}\left(\mathcal{F}_{i}, \mathcal{G}\right)$, in the derived category of the differential graded algebra $\bigoplus_{i j} \mathbf{R} \operatorname{Hom}\left(\mathcal{F}_{i}, \mathcal{F}_{j}\right)$, is faithful. (Here the algebra and module structures are the obvious ones; replacing each $\mathcal{F}_{i}$ by a finite resolution of injectives, which one can prove is possible, it is just composition of morphisms.)

In fact a difficult result of $[\mathbf{K S}]$ is that the induced action on homology, i.e. the action on the graded modules $\bigoplus_{i k} \operatorname{Ext}^{k}\left(\mathcal{F}_{i}, \mathcal{G}\right)$ in the derived category of the graded algebra $\bigoplus_{i j k} \operatorname{Ext}^{k}\left(\mathcal{F}_{i}, \mathcal{F}_{j}\right)$, is faithful. (Since the $\mathcal{F}_{i}$ s form an $A_{n}$-chain these modules and algebras take a standard form, and the braid group action is the one considered in $[\mathbf{K S}$.) However this is not enough to prove faithfulness of the action at the level of differential graded modules. The following result, though, is sufficient to provide a proof.

Theorem 6.1. For $\left\{\mathcal{F}_{i}\right\}_{i=1}^{n}$ an $A_{n}$-chain of $N$-spherical objects, $N \geq 2$, the graded algebra $A=\bigoplus_{i k} \operatorname{Ext}^{k}\left(\mathcal{F}_{i}, \mathcal{F}_{i}\right)$ is intrinsically formal. That is, any differential graded algebra with $A$ as its cohomology is quasi-isomorphic to $A$.

This is proved by a lot of non-commutative obstruction theory that I will not go into here. That such machinery is really necessary is seen in the following example of non-faithfulness in dimension $N=1$.

Let $T$ be an elliptic curve, $L$ be a non-trivial degree zero line bundle, and denote by $\mathcal{O}_{p}$ the structure sheaf of a point $p \in T$. Notice that $L$ induces an automorphism

$\phi_{L}$ of $T$ : identifying $T$ with its degree one line bundles, $\phi_{L}$ is given by tensoring with $L$.

The sheaves $\mathcal{O}=\mathcal{O}_{T}, \mathcal{O}_{p}$ and $L$ form an $A_{3}$-chain of 1-spherical sheaves.

THEOREM 6.2. The action of $T_{L}^{\prime} T_{\mathcal{O}}$ is the action induced by the automorphism $\phi_{L}$ by pullback. In particular if $L$ has order two $\left(L^{2}=\mathcal{O}\right)$ then $\left(T_{L}^{-1} T_{\mathcal{O}}\right)^{2} \cong \mathrm{id}$.

\section{References}

[AP] D. Arinkin and A. Polishchuk, Fukaya category and Fourier transform, preprint math/9811023.

[BO] A.I. Bondal and D. O. Orlov, Semiorthogonal decomposition for algebraic varieties, preprint alg-geom/9506012.

[Br] T. Bridgeland, Equivalences of triangulated categories and Fourier-Mukai transforms, Bull. Lond. Math. Soc. 31 (1999), 25-34.

[De] P. Deligne, Action du groupe des tresses sur une catégorie, Invent. Math. 128 (1997), 159-175.

[KS] M. Khovanov and P. Seidel, Quivers, Floer cohomology, and braid group actions, in preparation, 1999. 
[K] M. Kontsevich, Homological Algebra of Mirror Symmetry, International Congress of Mathematicians, Zürich 1994. Birkhäuser, 1995.

$[\mathrm{Ku}] \quad \mathrm{S} . \mathrm{A}$. Kuleshov, Exceptional bundles on K3 surfaces, in $\mathbf{R u}$.

[Ma] A. Maciocia, Generalized Fourier-Mukai transforms, J. Reine Angew. Math. 480 (1996), $197-211$.

[Mo] D. R. Morrison, Through the looking glass, Mirror Symmetry III (Montreal 1995), AMS/IP Stud. Adv. Math. 10 (1999), 263-277.

$[\mathrm{Mu}]$ S. Mukai, Duality between $D(X)$ and $D(\widehat{X})$ with its application to Picard sheaves, Nagoya Math. Jour. 81 (1981), 153-175.

[Or] D.O. Orlov, Equivalences of derived categories and K3 surfaces, preprint alggeom/9606006

[Pi] H. Pinkham, Singularités exceptionnelles, la dualité étrange d'Arnold, et les surfaces K3, C. R. Acad. Sc. Paris 284 A (1977) 615-618.

[Ru] A. N. Rudakov et al., Helices and vector bundles: Seminaire Rudakov, LMS Lecture Note Series 148, Cambridge University Press, 1990.

[S] P.S. Seidel, Lagrangian two-spheres can be symplectically knotted, preprint math/9803083.

[ST] P.S. Seidel and R. P. Thomas, Braid group actions on derived categories of sheaves, preprint 1999.

[SYZ] A. Strominger, S.-T. Yau and E. Zaslow, Mirror Symmetry is T-Duality, Nucl. Phys. B479 (1996), 243-259.

[Ty] A. N. Tyurin, Geometric quantization and mirror symmetry, preprint math.AG/9902027.

[Va] C. Vafa, Extending Mirror Conjecture to Calabi-Yau with Bundles, preprint hep-th/ 9804131.

Mathematical Institute, 24-29 St Giles', Oxford OX1 3LB. UK

E-mail address: thomas@maths.ox.ac.uk 